\title{
Die Emanzipation der Staatsanwaltschaft als Beitrag zur Unabhängigkeit der Justiz
}

\section{Ausgangspunkt}

Das Thema nimmt zwei Punkte vorweg. Nämlich dass sich der Staatsanwalt »in« der Justiz befindet, also Teil der Dritten Gewalt ist und ihr nicht als exekutives Kontrollorgan gegenüber steht. Und weiter, dass die Staatsanwaltschaft auch in einer selbstverwalteten Justiz eine Rolle spielen kann und nicht auf ministerielle Aufsicht und Leitung angewiesen ist.

\section{Bestimmung der Rolle der Staatsanwaltschaft in der Justiz}

Beides ist nicht selbstverständlich. Nach dem einschlägigen Amtsrecht sind Staatsanwaltschaften nicht nur nach der inneren Verfassung hierarchisch gegliederte Behörden, deren Angehörige den dienstlichen Anweisungen ihrer Vorgesetzten nachzukommen haben ${ }^{1}$. Sie unterstehen auch der Aufsicht und Leitung des jeweils zuständigen Justizministers ${ }^{2}$. Dieses »externe « Weisungsrecht ist umfassend; es ermächtigt zu allgemeinen Richtlinien und zu Weisungen im Einzelfall ${ }^{3}$. Im Bund und in einigen Bundesländern können die ersten Beamten jederzeit ohne Angabe von Gründen entlassen werden ${ }^{4}$ und sind auch in ihrem Status von der Exekutive abhängig. Die Justizminister üben diese Rechte zwar selten aus. Ihrer Bedeutung tut dies aber keinen Abbruch: Sie sind geeignet und ersichtlich auch dazu bestimmt, der Staatsanwaltschaft ein $»$ Wohlverhalten « gegenüber der Exekutive nahe zu legen ${ }^{5}$. Danach drängt sich der Eindruck einer weisungsunterworfenen Behörde auf, die - wie ein Glied der allgemeinen inneren Verwaltung - am Gängelband der Exekutive geht. In dieses düstere Bild passt eine jüngere Entscheidung des Bundesverfassungsgerichts, die die Staatsanwaltschaft trotz ihrer Eingliederung in die Justiz als Teil der Exekutive bezeichnet und von ihr keine strikte Neutralität erwartet, weil sie nicht unabhängig, sondern - wie die Polizei - ein weisungsunterworfenes Strafverfolgungsorgan sei $^{6}$. Dass eine Behörde, die derart weitgehenden Einflüssen der Exekutive unterliegt, sich in eine selbstverwaltete Justiz nicht einfügen könnte, sondern ihr gleichsam als Antagonist gegenüber stehen müsste, scheint mir auf der Hand zu liegen.

$1 \S 146$ GVG.

$2 \S 147$ Nr. 1, 2 GVG.

3 Markwardt, FS für Böttcher, S. 99; ob die externe Weisung an den einzelnen Staatsanwalt ergehen kann oder ob sie an den Generalstaatsanwalt zu richten ist, ist streitig.

$4 \S 36$ Abs. 1 Nr. 5 BBG; $\S 31$ Abs. 1 BRRG.

5 Bölter, FS Strauda, S. 300.

6 BVerfG NJW 2001, 1121, 1122f. 


\section{Verfassungsrechtlicher Rahmen für die Unabhängigkeit der Staatsanwaltschaft}

Nun wird vielfach behauptet, Aufsicht und Weisungsbefugnis der Justizverwaltungen seien von der Verfassung vorgegeben. Die Strafverfolgung müsste parlamentarisch verantwortet werden, deshalb dürfe im Bereich der Staatsanwaltschaften kein ministerialfreier Raum entstehen ${ }^{7}$. In dieser Allgemeinheit halte ich das nicht für richtig:

\section{Wortlaut}

Das Wort Staatsanwaltschaft oder Staatsanwalt ist im Verfassungstext nicht enthalten. Mit der Stellung der Staatsanwaltschaften befasst sich das Grundgesetz nicht. Keine Verfassungsnorm ordnet sie der Exekutive zu. Dass Art. 92 GG die Recht sprechende Gewalt den Richtern anvertraut, bedeutet nicht, dass jeder nicht richterliche staatliche Funktionsträger entweder Parlamentarier oder Angehöriger der Exekutive sein müsse ${ }^{8}$. Ersichtlich stellt es die Verfassung dem Gesetzgeber anheim, ob er überhaupt Staatsanwaltschaften einrichtet, mit welchen Aufgaben er sie betraut und ob er sie als Organe der Exekutive oder der Jurisdiktion ausgestaltet ${ }^{9}$. Sind sie Teil der Jurisdiktion, ist eine externe Aufsicht und Leitung nicht geboten; denn die dritte Gewalt ist nicht dem Parlament verantwortlich und sie ist per se »ministerialfrei«.

\section{Rechtsprechung des Bundesverfassungsgerichts}

Die bereits de lege lata ambivalente Stellung der Staatsanwaltschaft spiegelt sich in der Rechtsprechung des Bundesverfassungsgerichts wider. Das Gericht sieht sie als weisungsunterworfene Behörde aber auch als »wesentlichen Bestandteil der Justiz«, der mit den Gerichten an der Aufgabe der Justizgewährung mitwirkt ${ }^{10}$. Wenn eine jüngere Entscheidung zum Richtervorbehalt ${ }^{11}$ einseitig die Abhängigkeit von der Exekutive hervorhebt, dient dies nicht der Darstellung eines verfassungsrechtlichen Soll-Zustandes, sondern gibt die gegenwärtige Lage wieder, die durchaus kritisch gesehen wird. Auch dem bekannten Cannabisbeschluss des 2. Senats vom 9.3.1994 kann nicht entnommen werden, dass die Staatsanwaltschaft von Verfassungs wegen exekutiver Aufsicht unterstehen muss. Den Ländern wird dort zwar die Pflicht auferlegt, die staatsanwaltliche Einstellungspraxis bei Bagatellverstößen ${ }^{12}$ zu vereinheitlichen $^{13}$. Dabei kam es dem Gericht aber ganz offensichtlich auf die Gleichbehandlung, nicht auf die ministeriell verantwortete Weisung an. Externe Weisungen sind aber weder das einzige noch auch nur ein besonders effektives Mittel zur Durchsetzung einer gleichmäßigen Strafverfolgung. Den Justizministern ist es auf politischer Ebene nicht gelun-

7 Vgl. etwa Bölter, FS Strauda 293, 310ff. mit zahlreichen Nachweisen.

8 Dies würde verkennen, dass der Bereich der Rechtspflege über den der Rechtsprechung hinausreicht.

9 Anklage und Ermittlung waren bis in die Mitte des 19. Jhdt. Aufgabe des Richters.

10 BVerfGE 9, 223, 228f.

11 BVerfG NJW 2001, 1121.

$12 \S 31 \mathrm{a}$ BtMG betr. den Besitz von geringen Mengen zum Eigenverbrauch.

13 BVerfGE 90, 145, 190. 
gen, ihre Richtlinien zu vereinheitlichen ${ }^{14}$. Dass den professionell denkenden Generalstaatsanwälten ein Konsens schwerer fallen würde, erscheint mir nicht ausgemacht ${ }^{15}$.

\section{Keine Zuordnung der Staatsanwaltschaft zur Exekutive kraft Natur der Sache}

Strafverfolgung ist von der Natur der Sache nicht der Exekutive zuzuordnen. Aus der parlamentarischen Aufmerksamkeit, die die Tätigkeit der Staatsanwaltschaft bisweilen erregt $^{16}$, folgt solches nicht; nicht alles, was das Aufsehen der Öffentlichkeit erregt und zum Gegenstand parlamentarischer Erörterung werden kann, muss auch parlamentarisch verantwortet werden ${ }^{17}$. Dass die Staatsanwaltschaft bei ihren Ermittlungen Polizeikräfte in Anspruch nehmen muss, ist Folge des Fehlens eigener Kräfte. Diese organisatorische Zufälligkeit, die man übrigens bedauern mag, macht sie nicht zu einem Organ der Polizeiführung. Auch dort, wo die Polizei eingeschaltet wird, unterscheidet sich die ermittelnde Tätigkeit vom Verwaltungshandeln: Sie wird nicht vom Prinzip der Nützlichkeit regiert, sondern unterliegt den strengen rechtlichen Bindungen des Legalitätsprinzips, das über die Bindung der Verwaltung an Recht und Gesetz hinausgeht ${ }^{18}$. Soweit die Verfahrenseinstellung im Ermessen der Staatsanwaltschaft steht, handelt es sich nicht um das nicht näher strukturierte, vorwiegend an der Nützlichkeit ausgerichtete Entscheidungsspektrum, das die Verwaltungsbehörde etwa bei der Verfolgung von Ordnungswidrigkeiten beansprucht $(\$ 47$ Abs. $1 \mathrm{OWiG})$. Es handelt sich vielmehr um einen Spielraum, der in aller Regel ${ }^{19}$ nach justiztypischen Maßstäben auszufüllen ist. Meist geht es um geringe Schuld und fehlendes öffentliches Interesse an der Strafverfolgung also um Gegenstände, die typischerweise von der Dritten Gewalt zu bewerten sind.

\section{Zusammenfassung}

Zusammenfassend kann gesagt werden, dass die bestehenden Aufsichts- und Leitungsrechte der Justizverwaltung weder verfassungsrechtlich vorgegeben noch in der Natur der Sache angelegt sind. Verfassungswidrig ist der gegenwärtige Zustand allerdings nicht. Das Bundesverfassungsgericht erkennt vielmehr das Aufsichts- und Leitungsrecht der Landesjustizverwaltungen $\mathrm{an}^{20}$, und akzeptiert die durch das einfache Recht geschaffene Abhängigkeit von der Exekutive ${ }^{21}$. Mit anderen Worten: Zweifellos

14 Vgl. Hügel/Junge BtMG (Loseblattausgabe), § 31a, Rdn. 1, 2.

15 Vgl. Rautenberg, NJ 2003, 174.

16 Hund, ZRP 1994, 471f.

$17 \mathrm{Zu}$ denken ist etwa an sog. Skandalurteile.

18 BVerfGE 9, 223, 228; Hassemer (Strafen im Rechtsstaat, S. 67) sieht den Kern des Legalitätsprinzips darin, dass die StA die Strafverfolgung nicht von einer auf den Einzelfall bezogenen Folgenabwägung abhängig machen darf. § 46 I StGB steht dem nur scheinbar entgegen: Die Wirkungen der Strafe sind neben der Schuld nur sekundär zu berücksichtigen. Exekutives Denken würde demgegenüber das Ziel des Verwaltungshandelns und die Angemessenheit und Geeignetheit der Maßnahme zur Grundlage der Entscheidungsfindung machen.

19 Eine Ausnahme bilden die in der Praxis nahezu bedeutungslosen $\S 153 \mathrm{c}$ Abs. 3, 4 StPO.

20 So bereits BVerfGE 9, 223, 228; 90, 145, 190.

21 BVerfG NJW 2001, 1121. 
haben die Väter des Grundgesetzes gewusst, dass es neben unabhängigen Richtern auch weisungsgebundene Staatsanwälte gibt ${ }^{22}$; es fehlt aber jeglicher Anhaltspunkt dafür, dass sie den vorgefundenen Zustand für alle Zeiten zementieren wollten.

\section{Pro- und Contra-Erwägungen zum Leitbild eines »unabhängigen Staatsanwalts«}

Bei der Frage, ob dem Leitbild eines »unabhängigen Staatsanwalts « gefolgt werden soll, ist eine differenzierende Betrachtung angebracht.

\section{Folgen der Forderung nach persönlicher Unabhängigkeit der Staatsanwälte}

Die Forderung nach persönlicher Unabhängigkeit des Staatsanwalts würde die Struktur der Staatsanwaltschaften grundlegend in Frage stellen. Hierarchischer Aufbau und (interne) Weisungsbefugnis der ersten Beamten prägen das Bild der Staatsanwaltschaft. Sie sind unabdingbare Voraussetzung für die Herstellung einer gleichmäßigen Strafrechtspflege und stellen insoweit das notwendige organisatorische Pendant zur richterlichen Unabhängigkeit dar: Dem individuell unabhängigen Richter steht die hierarchisch geordnete Staatsanwaltschaft als Kontrollbehörde gegenüber. Die Notwendigkeit des internen Weisungsrechts wird im Grundsatz nicht bezweifelt; für eine selbstverwaltete und deshalb »ministerialfreie « Justiz wird das nicht in minderem Maße gelten. Der Cannabisbeschluss des Bundesverfassungsgerichts fordert von Verfassungs wegen die Vereinheitlichung der staatsanwaltlichen Einstellungspraxis ${ }^{23}$; er setzt damit eine einheitliche Leitung voraus. Folgt man dieser Konzeption ist Unabhängigkeit ein Attribut, das allenfalls der Behörde der Staatsanwaltschaft, nicht aber dem einzelnen Staatsanwalt zukommen kann. Damit soll nicht gesagt sein, dass das interne Weisungsrecht nicht reformbedürftig wäre und keine Einschränkungen vertrüge $^{24}$. Seine ersatzlose Abschaffung würde indessen einen Systembruch bedeuten.

\section{Der Begriff des Staatsanwalts als »politischer Beamter"}

Im Bund und in einigen wenigen Ländern können die ersten Beamten der Staatsanwaltschaft als sog. politische Beamte ${ }^{25}$ jederzeit in den einstweiligen Ruhestand versetzt werden. Nach der Legaldefinition des $\S 31$ Abs. 1 BRRG bekleidet der politische Beamte ein Amt, bei dessen Ausübung er in fortdauernder Übereinstimmung mit den grundsätzlichen politischen Ansichten und Zielen der Regierung stehen muss. Beim Generalbundesanwalt ist dies zu vermitteln: Die Zuständigkeit für den Staatsschutz impliziert einen (auch) politisch zu verantwortenden Umgang mit dem Strafrecht; diese

22 Vgl. Sarstedt, NJW 1964, 1752, 1755.

23 BVerfGE 90, 145, 190; divergierende richterliche Entscheidungen werden als notwendige Folge der Unabhängigkeit hingenommen.

24 Insoweit liegt ein bedenkenswerter Gesetzesentwurf der Staatsanwaltskommission des deutschen Richterbundes vor; vgl. hierzu Hannich, DRiZ 2003, 249.

$25 \S 36$ Abs. 1 Nr. 5 BBG; § 31 Abs. 1 BRRG. 
Besonderheit wird durch das Gesetz anerkannt (vgl. etwa $\$ 153 \mathrm{~d} \mathrm{StPO}{ }^{26}$ ). Auf das Amt der Generalstaatsanwälte in den Ländern passt der politische Beamte aber nicht: Strafverfolgung hat das Recht und nicht die politischen Ansichten und Ziele der jeweiligen Regierung durchzusetzen ${ }^{27}$. Die Öffentlichkeit hat dafür ein feines Gespür: Die Abberufung von Generalstaatsanwälten wurde nahezu durchgängig als Übergriff der Exekutive wahrgenommen ${ }^{28}$; dem Vertrauen in die Integrität der Staatsanwaltschaft war solches nicht förderlich. Diese Einsicht scheint sich zu verbreiten: Seit 2000 haben die Länder Nordrhein-Westfalen, Berlin und Thüringen ihre Generalstaatsanwälte »entpolitisiert«. Die übrigen Bundesländer sind dem Vernehmen nach im Begriff, dies ebenfalls zu tun.

\section{Weisungsbefugnisse des Justizministers}

Die Weisungsbefugnis des Justizministers ist in Bezug auf den Generalbundesanwalt verständlich und dadurch bedingt, dass der Schwerpunkt seines Aufgabenbereichs im Schnittpunkt von Strafverfolgung und Politik liegt. Bei den Landesstaatsanwaltschaften steht das externe Weisungsrecht hingegen in einem Spannungsverhältnis zu den historisch gewachsenen staatsanwaltlichen Aufgaben. Bekanntlich wurden die Staatsanwaltschaften als Wächterinnen des Gesetzes zunächst in Preußen und sodann im Deutschen Reich eingeführt. Sie sollten dem Recht Genüge verschaffen und zwar gleichermaßen im Interesse der Strafverfolgung wie im Interesse des Angeklagten. Deshalb wurden ihnen Kontrollbefugnisse und Kontrollfunktionen gegenüber Gerichten und Polizei zuerkannt ${ }^{29}$. Die Staatsanwaltschaft besitzt das Anklagemonopol; in der Strafrechtspflege hängt die Justizgewährung weitgehend von ihrer Entschließung $\mathrm{ab}^{30}$. Dass eine weisungsabhängige Behörde über diese Aufgabe allzu leicht in Zielkonflikte gerät, leuchtet unmittelbar ein. Das Legalitätsprinzip legt den Staatsanwaltschaften eine strenge, nicht am gewünschten Ergebnis, sondern am vorgefundenen Tatbestand orientierte Gesetzesbindung auf. Dies gedeiht am besten im Klima einer strikten Neutralität, die das Bundesverfassungsgericht von einem verlängerten Arm der Exekutive gerade nicht erwartet ${ }^{31}$. Und in der Tat: Eine Staatsanwaltschaft, die wie eine höhere Polizeiführung exekutiver Aufsicht und Leitung untersteht, verschafft dem Ermittlungsverfahren nur einen beschränkten rechtsstaatlichen Mehrwert. Die immer intensiver werdende ermittlungsrichterliche Kontrolle staatsanwaltlichen Handelns unterstreicht dies. Dass die preußische Staatsanwaltschaft den Weisungen des Justizministers unterstand ${ }^{32}$, musste einem Staat, in dem die Justiz durch die Krone ho-

26 Die Voraussetzungen des Absehens von der Strafverfolgung nach dieser Vorschrift (Gefahr eines schweren Nachteils für die Bundesrepublik Deutschland oder das Entgegenstehen sonstiger überwiegender öffentlicher Interessen) sind im Grunde nicht rechtlicher, sondern politischer Natur (vgl. die Beispiele bei Müller/Wach, Rebmann FS, 339f.).

27 Roxin, DRiZ 1997, 109.

28 Rautenberg, DRiZ 2000, 141.

29 Vgl. Schaefer, NJW 2001, 1396.

30 Hannich, DRiZ 2003, 249, 253.

31 BVerfG NJW 2001, 1121, 1122.

32 Bölter, Strauda FS, S. 297; Hund, ZRP 1994, 470f.; zum württembergischen Gegenbeispiel vgl. Günter, DRiZ 2002, 55. 
heitlich gewährt wurde, noch selbstverständlich erscheinen. Für die Staatsanwaltschaft in der modernen rechtsstaatlichen Demokratie sollten daraus keine Folgen gezogen werden.

\section{Schluss}

Das derzeitige Amtsrecht, das die Staatsanwaltschaft den externen Weisungen der Justizverwaltungen unterwirft und die jederzeitige Ablösung von Generalstaatsanwälten zulässt, ist mit einer selbst verwalteten Justiz nicht zu vereinen. Bereits jetzt steht es in einem Spannungsverhältnis zu der nach geltendem Recht bestehenden Eingliederung der Staatsanwaltschaft in die Justiz, ohne allerdings - legt man die Rechtsprechung des Bundesverfassungsgerichts zugrunde - verfassungswidrig zu sein. Die bestehenden Aufsichts- und Leitungsrechte der Justizverwaltung sind von Verfassungs wegen nicht geboten und damit durch den einfachen Gesetzgeber gestaltbar. Mit Ausnahme des Generalbundesanwalts, für den wegen seiner Zuständigkeit für Staatsschutzstrafsachen besondere Regeln gelten, ist die strafverfolgende Tätigkeit des Staatsanwalts kein Gegenstand der zwingend ministeriell oder parlamentarisch verantwortet werden müsste. sondern eine Aufgabe, die eher der Justiz zugeordnet ist. Es steht dem Gesetzgeber frei, die amtsrechtliche Konsequenz zu ziehen, indem er die Staatsanwaltschaft von externen Weisungen frei stellt. Er wäre gut beraten, wenn er diesen Spielraum nutzen würde. Der Blick über den Zaun zeigt, dass Deutschland, was die Emanzipation seiner Staatsanwaltschaften angeht, hinter der Entwicklung herhinkt. 